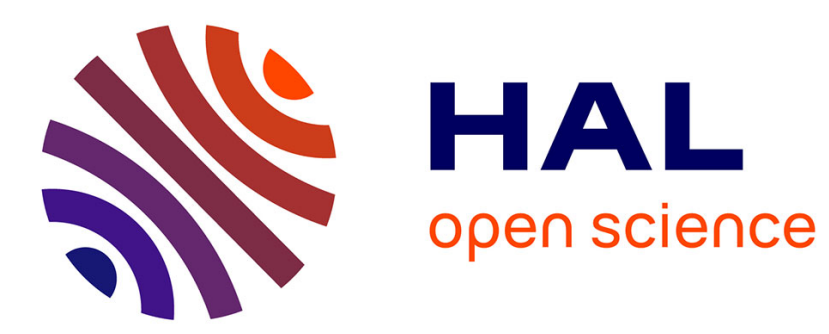

\title{
Homogeneous Differentiator Design using Implicit Lyapunov Function Method
}

\author{
Andrey Polyakov, Denis Efimov, Wilfrid Perruquetti
}

\section{To cite this version:}

Andrey Polyakov, Denis Efimov, Wilfrid Perruquetti. Homogeneous Differentiator Design using Implicit Lyapunov Function Method. European Control Conference, IEEE, IFAC, Jun 2014, Strasbourg, France. hal-01003992

\section{HAL Id: hal-01003992 \\ https://hal.inria.fr/hal-01003992}

Submitted on 11 Jun 2014

HAL is a multi-disciplinary open access archive for the deposit and dissemination of scientific research documents, whether they are published or not. The documents may come from teaching and research institutions in France or abroad, or from public or private research centers.
L'archive ouverte pluridisciplinaire HAL, est destinée au dépôt et à la diffusion de documents scientifiques de niveau recherche, publiés ou non, émanant des établissements d'enseignement et de recherche français ou étrangers, des laboratoires publics ou privés. 


\title{
Homogeneous Differentiator Design using Implicit Lyapunov Function Method
}

\author{
Andrey Polyakov, Denis Efimov, Wilfrid Perruquetti
}

\begin{abstract}
The Implicit Lyapunov Function (ILF) for a class of homogeneous systems is introduced and studied. The analysis of homogeneous differentiator using ILF method is presented. Sufficient stability conditions for homogeneous differentiator are obtained and represented by a parameterized system of Linear Matrix Inequalities (LMI). The differentiation error and convergence time are estimated. The procedure of parameters tuning for homogeneous differentiator is formulated as the semi-definite programming problem with LMI constraints. The obtained theoretical results are supported by numerical simulations.
\end{abstract}

\section{INTRODUCTION}

Differentiators are important for many applications, e.g. PID controllers implementation [1], system states observation [2], [3], designing of fault detection algorithms [4], digital filter in signal processing [5], identification [6], etc.

From theoretical point of view, the differentiators can provide the exact values of the signal's derivatives (see, for example, [7], [8]). It is worth to stress that any exact differentiator is a mathematical abstraction, since, in practice, due to measurement noises and finite computational precision of digital devices it will always give approximative solutions only.

The present paper studies the so-called homogeneous differentiator [9]. The well-known linear high-gain differentiator [2] and exact high-order sliding mode (HOSM) differentiator [8] can be obtained as partial cases of the homogeneous differentiator, when the homogeneity degree [9] tends to 0 and -1 , respectively.

In contrast to linear high-gain differentiator [2] [10], the effective schemes for parameters tuning of HOSM differentiators and homogeneous differentiators are not well developed for high order cases. Several papers are devoted to a design of high-order homogeneous observers (differentiators) (see, for example, [8], [9]). The developed methods just guarantee the existence of the appropriate observers' parameters.

*This work was supported by EU Interreg IV A 2 Mers Seas Zeeën Cross-border Cooperation Programme under SYSIASS project 06-020.

Andrey Polyakov, Denis Efimov, Wilfrid Perruquetti are with Non-A INRIA - LNE, Parc Scientifique de la Haute Borne 40, avenue Halley Bat.A, Park Plaza 59650 Villeneuve d'Ascq(e-mail: andrey.polyakov@inria.fr, denis.efimov@inria.fr, wilfrid.perruetti@inria.fr). They are also with LAGIS UMR CNRS 8219, Ecole Centrale de Lille, BP 48, Cité Scientifique, 59651 Villeneuve-d'Ascq, France.

Denis Efimov is also with Department of Control Systems and Informatics, National Research University ITMO, 49 Kronverkskiy av., 197101 Saint Petersburg, Russia.

This work was supported in part by the Government of Russian Federation (Grant 074-U01) and the Ministry of Education and Science of Russian Federation (Project 14.Z50.31.0031).
The present paper addresses the problem of the homogeneous differentiator design using the Implicit Lyapunov Function (ILF) method [11], [12], [13]. The homogeneous ILF allows us to prove the finite-time convergence of homogeneous differentiator and to present the simple constructive scheme for its parameters tuning, which is based on the semidefinite programming technique with LMI constraints.

The paper is organized as follows. The next section presents the notation to be used. The section III discuss the problem statement. Then some supporting facts related to homogeneity, finite-time stability and ILF method are presented. The structure of homogeneous implicit Lyapunov function is studied. Next, the main results of the paper are formulated. Finally, the numerical simulation results and conclusions are given. Proof of the theorem about homogeneous implicit Lyapunov function is presented in the Appendix.

\section{NOTATION}

- $\mathbb{R}_{+}=\{x \in \mathbb{R}: x>0\}$, where $\mathbb{R}$ is the set of real numbers.

- The positive (negative) definiteness (semi-definiteness) of a symmetric matrix $P=P^{T} \in \mathbb{R}^{n \times n}$ is denoted by $P>0(<0, \leq 0, \geq 0)$.

- Let us denote the square root of the matrix $P>0$ by $P^{\frac{1}{2}}$, i.e. $P^{\frac{1}{2}}=Q>0$ such that $Q^{2}=P$.

- Let $\lambda_{\min }(P)$ and $\lambda_{\max }(P)$ be the minimum and maximum eigenvalue of a symmetric matrix $P=P^{T} \in$ $\mathbb{R}^{n \times n}$, respectively.

- For $z=\left(z_{1}, \ldots, z_{n}\right)^{T} \in \mathbb{R}^{n}$ the operator $\operatorname{diag}(z)$ denotes the diagonal matrix with the diagonal elements $z_{i}, \operatorname{diag}\left\{z_{i}\right\}_{i=1}^{n}=\operatorname{diag}(z)$.

- $\mathcal{C}^{k}$ is the set of functions $\mathbb{R} \rightarrow \mathbb{R}$, which have continuous derivatives at least up to the order $k$. If $\xi \in \mathcal{C}^{k}$ then $\xi^{(l)}$ denotes $l$-th derivative of $\xi$ for $l \leq k$.

- A continuous function $\alpha: \mathbb{R}_{+} \rightarrow \mathbb{R}_{+}$belongs to the class $\mathcal{K}$ if $\alpha(0)=0$ and the function is strictly increasing.

\section{PROBLEM STATEMENT}

Consider the system

$$
\left\{\begin{array}{l}
\dot{x}=A x+b f(t, x) \\
y=x_{1}
\end{array}\right.
$$

where

- $x=\left(x_{1}, x_{2}, \ldots, x_{n}\right)^{T} \in \mathbb{R}^{n}$ is the system state,

- $y \in \mathbb{R}$ is the system output, which can be measured on-line, 


$$
A=\left(\begin{array}{ccccc}
0 & 1 & 0 & \ldots & 0 \\
0 & 0 & 1 & \ldots & 0 \\
\ldots & \ldots & \ldots & \ldots & \ldots \\
0 & 0 & 0 & \ldots & 1 \\
0 & 0 & 0 & \ldots & 0
\end{array}\right), \quad b=(0,0, \ldots, 1)^{T},
$$

- $f: \mathbb{R} \times \mathbb{R}^{n} \rightarrow \mathbb{R}$ is an unknown bounded function

$$
|f(t, x)| \leq f_{0}, \quad \text { for } \forall(t, x) \in \mathbb{R}^{n+1},
$$

where $f_{0}$ is a known positive number.

The problem is to observe the whole state vector $x(t)$ of the system (1) using the output measurements $y(t)$.

If $y(t)=\xi(t) \in \mathcal{C}^{n+1}$ and $f(t, x)=\xi^{(n+1)}(t)$ then the observation problem for the system (1) is just the differentiation of the signal $\xi$. Indeed, in this case we have $x_{i}(t)=\xi^{(i-1)}(t)$.

\section{PRELIMINARIES}

\section{A. Finite-time Stability and Homogeneity}

Consider the system of the form

$$
\dot{x}=f(x), \quad x(0)=x_{0},
$$

where

- $x \in \mathbb{R}^{n}$ is the state vector,

- $f: \mathbb{R}^{n} \rightarrow \mathbb{R}^{n}$ is a nonlinear continuous vector field.

Assume that the origin is an equilibrium point of the system (3), i.e. $f(0)=0$.

Definition 1 ([14], [15]): The origin of system (3) is said to be globally finite-time stable (FTS) if:

1) Finite-time attractivity: there exists a function $T$ : $\mathbb{R}^{n} \backslash\{0\} \rightarrow \mathbb{R}_{+}$, such that for any $x_{0} \in \mathbb{R}^{n} \backslash\{0\}$, $\lim _{t \rightarrow T\left(x_{0}\right)} x\left(t, x_{0}\right)=0$.

2) Lyapunov stability: there exists a function $\delta \in \mathcal{K}$ such that for all $x_{0} \in \mathbb{R}^{n},\left\|x\left(t, x_{0}\right)\right\| \leq \delta\left(\left\|x_{0}\right\|\right)$.

The function $T$ is called the settling-time function of the system (3).

Let $\lambda, r_{i} \in \mathbb{R}_{+}, i \in\{1, \ldots, n\}$ then one can define:

- the vector of weights $r=\left(r_{1}, \ldots, r_{n}\right)^{T}$,

- the dilation matrix

$$
D_{r}(\lambda)=\operatorname{diag}\left\{\lambda^{r_{i}}\right\}_{i=1}^{n},
$$

note that $D_{r}(\lambda) x=\left(\lambda^{r_{1}} x_{1}, \ldots, \lambda^{r_{i}} x_{i}, \ldots, \lambda^{r_{n}} x_{n}\right)^{T}$.

Definition 2: [16] Set $r=\left(r_{1}, \ldots, r_{n}\right)$ with $r_{i} \in \mathbb{R}_{+}$. A function $g: \mathbb{R}^{n} \rightarrow \mathbb{R}$ (resp. a vector field $f: \mathbb{R}^{n} \rightarrow \mathbb{R}^{n}$ ) is said to be $r$-homogeneous of degree $m$ iff for all $\lambda \in \mathbb{R}_{+}$ and for all $x \in \mathbb{R}^{n}$ we have

$$
\lambda^{-m} g\left(D_{r}(\lambda) x\right)=g(x)
$$

$$
\text { (resp. } \left.\lambda^{-m} D_{r}^{-1}(\lambda) f\left(D_{r}(\lambda) x\right)=f(x)\right) \text {. }
$$

For a given $x \in \mathbb{R}^{n}$, the set

$$
\Gamma(x)=\left\{z \in \mathbb{R}^{n}: z=D_{r}(\lambda) x, \lambda \in \mathbb{R}_{+}\right\}
$$

is a curve on $\mathbb{R}^{n}$ (see, Fig. 1). An object is homogeneous iff the behavior of this object is symmetric along these particular curves.
Theorem 3 ([17], Proposition 6.1 and Theorem 7.1):

Let $f: \mathbb{R}^{n} \rightarrow \mathbb{R}^{n}$ be defined on $\mathbb{R}^{n}$ and be a continuous $\mathbf{r}$-homogeneous vector field with a negative degree. If the origin of the system (3) is locally attractive then it is globally finite time stable.

\section{B. Homogeneous Implicit Lyapunov Function}

The theorem given below provides the background for finite-time stability analysis of the nonlinear system (3) using implicit Lyapunov function.

Theorem 4: [13] Let there exists a continuous function $Q: \mathbb{R}_{+} \times \mathbb{R}^{n} \rightarrow \mathbb{R}$

such that

$$
(V, x) \mapsto Q(V, x)
$$

C1) $Q$ is continuously differentiable $\forall x \in \mathbb{R}^{n} \backslash\{0\}$ and $\forall V \in$ $\mathbb{R}_{+}$;

C2) for any $x \in \mathbb{R}^{n} \backslash\{0\}$ there exist $V^{-} \in \mathbb{R}_{+}$and $V^{+} \in$ $\mathbb{R}_{+}$:

$$
Q\left(V^{-}, x\right)<0<Q\left(V^{+}, x\right)
$$

C3) for $\Omega=\left\{(V, x) \in \mathbb{R}^{n+1}: Q(V, x)=0\right\}$ we have $\lim _{\substack{x \rightarrow 0 \\(V, x) \in \Omega}} V=0^{+}, \lim _{\substack{V \rightarrow 0^{+} \\(V, x) \in \Omega}}\|x\|=0, \lim _{\substack{\|x\| \rightarrow \infty \\(V, x) \in \Omega}} V=+\infty ;$

C4) the inequality

$$
-\infty<\frac{\partial Q(V, x)}{\partial V}<0
$$

holds $\forall V \in \mathbb{R}_{+}$and $\forall x \in \mathbb{R}^{n} \backslash\{0\}$;

C5) the inequality

$$
\frac{\partial Q(V, x)}{\partial x} f(x) \leq c V^{1-\mu} \frac{\partial Q(V, x)}{\partial V}
$$

holds $\forall(V, x) \in \Omega$, where $c>0$ and $0<\mu<1$ are some constants.

Then the origin of system (3) is globally finite-time stable with the following settling time estimate $T\left(x_{0}\right) \leq \frac{V_{0}^{\mu}}{c \mu}$, where $V_{0} \in \mathbb{R}_{+}: Q\left(V_{0}, x_{0}\right)=0$.

The proof of this theorem is based on the classical Implicit Function Theorem [18] providing $\frac{\partial V}{\partial x}=-\left[\frac{\partial Q}{\partial V}\right]^{-1} \frac{\partial Q}{\partial x}$. The condition C5) implies $\dot{V}(x) \leq-c V^{1-\mu}(x)<0$, where $V: \mathbb{R}^{n} \rightarrow \mathbb{R}_{+}$is a function implicitly defined by the algebraic equation $Q(V, x)=0$. The conditions $C 1)-C 4)$ of Theorem 4 just guarantee that the corresponding function $V$ is proper (continuously differentiable outside the origin, positive definite and radially unbounded).

The linear system is the simplest example of the system with homogeneous vector field. The positive definite quadratic form is the standard Lyapunov function of a stable linear system. Any level set of the quadratic Lyapunov function is an ellipsoid. Let us design the Lyapunov function for a homogeneous system (3) providing the same geometric property to a level set.

Introduce the following ILF candidate [19], [13]

$$
Q(V, x):=x^{T} D_{r}\left(V^{-1}\right) P D_{r}\left(V^{-1}\right) x-1,
$$

where $V \in \mathbb{R}_{+}, x \in \mathbb{R}^{n}$,

- $D_{r}(\cdot)$ is the dilation matrix of the form (4) with $r=$ $\left(r_{1}, r_{2}, \ldots, r_{n}\right)^{T} \in \mathbb{R}_{+}^{n}$; 


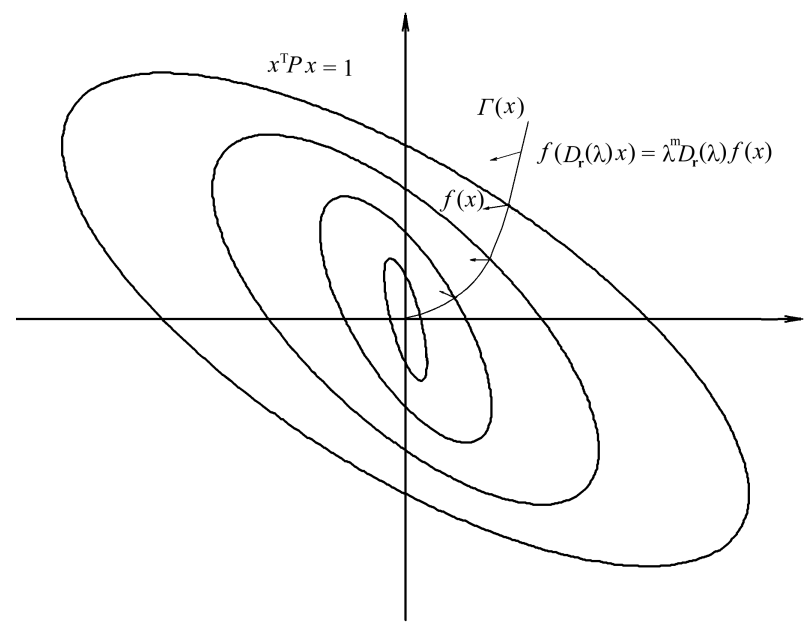

Fig. 1. Level lines of ILF and homogeneity curve $\Gamma(x)$

- $P \in \mathbb{R}^{(n+1) \times(n+1)}$ is a symmetric positive definite matrix.

Theorem 5 given below has the simplest geometric interpretation: if the homogeneous system (3) admits a Lyapunov function with at least one ellipsoidal level set, then it has a homogeneous ILF defined by the formula (5). The level lines of such Lyapunov function for $n=2$ are illustrated by Fig. 1.

\section{Theorem 5: If}

1) the continuous vector field $f: \mathbb{R}^{n} \rightarrow \mathbb{R}^{n}$ is $r$ homogeneous of degree $m$ with the vector of weights $r=$ $\left(r_{1}, r_{2}, \ldots, r_{n}\right)^{T} \in \mathbb{R}_{+}^{n}$,

2) the matrix $P>0$ satisfy the matrix inequality

$$
\operatorname{diag}(r) P+P \operatorname{diag}(r)>0,
$$

3) the inequality

$$
y^{T} P f(y)+f^{T}(y) P y<0
$$

holds for any $y \in \mathbb{R}^{n}: y^{T} P y=1$,

then the equation $Q(V, x)=0$ with $Q$ given by (5) implicitly defines the homogeneous Lyapunov function for the system (3) and

a) for $m>0$ the system (3) is asymptotically stable,

b) for $m<0$ the system (3) is finite-time stable and the settling time function $T: \mathbb{R}^{n} \rightarrow \mathbb{R}_{+} \cup\{0\}$ admits the following estimate:

$$
T\left(x_{0}\right) \leq \frac{\gamma V_{0}^{-m}}{\alpha}
$$

where

$$
\begin{gathered}
V_{0} \in \mathbb{R}_{+}: Q\left(V_{0}, x_{0}\right)=0 \text { for } x_{0} \neq 0, \\
\gamma=\lambda_{\max }\left(P^{-\frac{1}{2}} \operatorname{diag}(r) P^{\frac{1}{2}}+P^{\frac{1}{2}} \operatorname{diag}(r) P^{-\frac{1}{2}}\right), \\
\alpha=\max _{y \in \mathbb{R}^{n}: y^{T} P y=1}\left|y^{T} P f(y)+f^{T}(y) P y\right|,
\end{gathered}
$$

c) for $m=0$ the system (3) is exponentially stable:

$$
\|x(t)\| \leq \frac{\max \left\{V_{0}^{r_{\min }}, V_{0}^{r_{\max }}\right\}}{\sqrt{\lambda_{\min }(P)}} e^{-\frac{r_{\min \alpha}}{\gamma} t}
$$

where $r_{\min }=\min _{i=1,2, \ldots, n} r_{i}, r_{\max }=\min _{i=1,2, \ldots, n} r_{i}$.

The proof of this theorem is given in the appendix.

\section{ILF ANALysis of Homogeneous DifFERENTIATOR}

Consider the following observer

$$
\frac{d}{d t} \hat{x}=\left(A+D_{\tilde{r}}^{-1}\left(V\left(\left(\hat{x}_{1}-y\right) h\right)\right) k h^{T}\right)(\hat{x}-y h)
$$

where

- $y \in \mathbb{R}$ is an output of the system (1),

- $\hat{x}=\left(\hat{x}_{1}, \hat{x}_{2}, \ldots, \hat{x}_{n}\right) \in \mathbb{R}^{n}$ is the observer state,

- $k=\left(k_{1}, \ldots, k_{n}\right)^{T} \in \mathbb{R}^{n}, h=(1,0, \ldots, 0)^{T} \in \mathbb{R}^{n}$ os the vector of the gains,

- $D_{\tilde{r}}(\cdot)$ is the dilation matrix of the form (4) with

$$
\tilde{r}=(\mu, 2 \mu, \ldots, n \mu)^{T}, 0 \leq \mu \leq 1,
$$

- the positive definite function $V(z)$ is implicitly defined by the equation $Q(V, z)=0$ for $Q(V, z)$ defined by (5) with a positive definite matrix $P=P^{T} \in \mathbb{R}^{n \times n}$ and

$$
r=(1+(n-1) \mu, 1+(n-2) \mu, \ldots, 1+\mu, 1)^{T} \in \mathbb{R}^{n} .
$$

Remark 6: The structure of the equation $Q(V, z)=0$ implies

$$
V\left(\left(\hat{x}_{1}-y\right) h\right)=\left|\hat{x}_{1}-y\right|^{1 /(1+(n-1) \mu)} p_{11}^{1 /(2+2(n-1) \mu)},
$$

where $p_{11}=h^{T} P h$. So, the system (8) is, in fact, the homogeneous differentiator [9]:

$$
\begin{aligned}
& \frac{d \hat{x}_{1}}{d t}=\tilde{k}_{1}\left|\hat{x}_{1}-y\right|^{1-\mu /(1+(n-1) \mu)} \operatorname{sign}\left[\hat{x}_{1}-y\right]+\hat{x}_{2}, \\
& \frac{d \hat{x}_{2}}{d t}=\tilde{k}_{2}\left|\hat{x}_{1}-y\right|^{1-2 \mu /(1+(n-1) \mu)} \operatorname{sign}\left[\hat{x}_{1}-y\right]+\hat{x}_{3}, \\
& \cdots \\
& \frac{d \hat{x}_{n}}{d t}=\tilde{k}_{n}\left|\hat{x}_{1}-y\right|^{1-n \mu /(1+(n-1) \mu)} \operatorname{sign}\left[\hat{x}_{1}-y\right],
\end{aligned}
$$

where $\tilde{k}_{i}=k_{i} p_{11}^{-i \mu /(2+2(n-1) \mu)}, i=1,2, \ldots, n$.

If $\mu=1$, then the system (8) takes the form of the highorder sliding mode differentiator [8]. For $\mu=0$ it is a conventional linear observer.

Introduce the parameterized family of ellipsoids

$$
\varepsilon(\lambda, r, P):=\left\{z \in \mathbb{R}^{n}: z^{T} D_{r}\left(\lambda^{-1}\right) P D_{r}\left(\lambda^{-1}\right) z \leq 1\right\} .
$$

where $\lambda \in \mathbb{R}_{+}, P \in \mathbb{R}^{n \times n}$ is positive definite matrix and $D_{r}(\cdot)$ is the dilation matrix with $r$ defined by (4).

Theorem 7: Let for some $\alpha, \beta \in \mathbb{R}_{+}: \alpha>\beta$ and $\delta, \mu \in$ $(0,1)$ the system of matrix inequalities $(6)$ and

$$
\begin{aligned}
& \left(\begin{array}{cc}
P\left(A+k h^{T}\right)+\left(A+k h^{T}\right)^{T} P & \sqrt{f_{0}} P b \\
+(\alpha+\beta) P & -1
\end{array}\right) \leq 0, \\
& P>0, \quad P \geq \delta \operatorname{diag}(h) P \operatorname{diag}(h) \\
& \frac{k^{T}\left(I_{n}-D_{\tilde{r}}(\lambda)\right) D_{r}(\lambda) P D_{r}(\lambda)\left(I_{n}-D_{\tilde{r}}(\lambda)\right) k}{\beta^{2} \lambda^{2 \mu} h^{T} P h} \leq 1, \\
& \forall \lambda \in\left[0, \lambda^{*}\right], \quad \lambda^{*}=\delta^{-1 /(2+2(n-1) \mu)}
\end{aligned}
$$

is feasible for $P \in \mathbb{R}^{n \times n}, k \in \mathbb{R}^{1 \times n}$ with $\tilde{r}, r$ defined by (9), (10), respectively. Then 
1) for $f_{0} \neq 0$ any solution of the system (8) satisfies the relation

$$
(\hat{x}(t)-x(t)) \rightarrow \varepsilon\left(V_{*}, r, P\right) \quad \text { if } t \rightarrow+\infty,
$$

where $V_{*}=\left(f_{0} /(\alpha-\beta)\right)^{0.5 /(1-\mu)}$;

2) for $f_{0}=0$ any solution of the system (8) converges to $x(t)$ in a finite time, i.e. $\hat{x}(t)=x(t)$ for $t \geq T(\hat{x}(0)-x(0))$, and the settling-time function has the following estimate

$$
T(z) \leq \frac{\gamma V_{z}^{\mu}}{\alpha-\beta},
$$

where $V_{z} \geq 0: Q\left(V_{z}, z\right)=0$ and

$$
\gamma>0: \gamma P \geq P H_{r}+H_{r} P>0.1
$$

The proof of the main theorem is skipped due to space restrictions.

In order to apply the results of the presented theorem in practice we need to resolve the parameterized system of nonlinear matrix inequalities (13)-(15) with respect to variables $P$ and $k$ for some positive scalars $\alpha, \beta, \mu$ and $\delta$. In general case this is quite a complex problem, since the mentioned system of matrix inequalities is nonlinearly depended on the scalar parameter $\lambda$. The algorithms for resolving of parametric LMIs presented in [20] can be used. More simple scheme can also be presented for practical selection of observer parameters.

Proposition 8: Let for some fixed $\alpha, \beta \in \mathbb{R}_{+}: \alpha>\beta$ and $\mu \in(0,1)$ the system of linear matrix inequalities

$$
\begin{gathered}
\left(\begin{array}{cc}
\tilde{P} A+\tilde{y} h^{T}+A^{T} \tilde{P}+h \tilde{y}^{T} & -\sqrt{f_{0}} \tilde{P} b \\
+(\alpha+\beta) \tilde{P} & -q
\end{array}\right) \leq 0, \\
-\sqrt{f_{0}} b^{T} \tilde{P} \\
\tilde{P}>0, \quad \tilde{P} H_{r}+H_{r} \tilde{P}>0, \\
\tilde{P} \geq \delta \operatorname{diag}(h), \quad 0<\delta<1, \\
\left(\begin{array}{cc}
\gamma \beta^{2} & \tilde{y}^{T} \\
\tilde{y} & \tilde{P}
\end{array}\right) \geq 0, \quad h^{T} \tilde{P} h=1, \quad \gamma>0, \quad q>0
\end{gathered}
$$

is feasible for $\tilde{P}=\tilde{P}^{T} \in \mathbb{R}^{n \times n}, \tilde{y} \in \mathbb{R}^{1 \times n}$, then the matrix $P=\tilde{P} / q$ and the vector $k=\tilde{P}^{-1} \tilde{y}$ satisfy the system of matrix inequalities (6), (13)-(14).

The proof of this proposition is trivial. The condition (18) implies $\tilde{P}_{11}=1$. So, defining $p_{11}=1 / q>0$ and $P=\tilde{P} / q$ provides that (16)-(17) are equivalent to (13)-(14).

Since the pair $(A, h)$ is observable then the system of linear matrix inequalities (16) - (18) is always feasible at least for sufficiently small $f_{0}$ and $\mu$.

Using the Shur complement the LMI (18) can be equivalently rewritten in the form

$$
\beta^{2} h^{T} P h \geq \frac{1}{\gamma} k^{T} P k .
$$

Hence, in order to guarantee fulfilling the parametric matrix inequality (15) the parameter $\gamma$ has to be minimized under the LMI constraints (16)-(18). On the other hand, since $\left\|\lambda^{-\mu}\left(I_{n}-D_{\tilde{r}}(\lambda)\right) D_{r}(\lambda)\right\|$ tends to infinity as $\lambda \rightarrow+\infty$, then for the same reason we need to maximize the parameter $\delta$ (i.e. minimize $\lambda^{*}$ ).

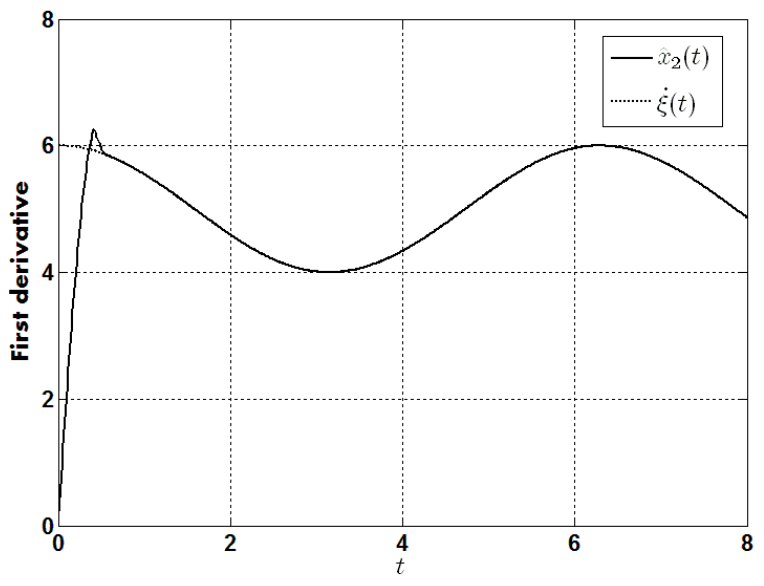

Fig. 2. The first derivative of the reference signal

Therefore, based on the given proposition the following heuristic scheme for tuning of control parameters can be introduced:

$$
\begin{gathered}
\min _{\delta, \gamma, q, \tilde{y}, \tilde{P}} \gamma-\delta \\
\text { subject to }(16)-(18) .
\end{gathered}
$$

When the optimal solution of this problem is found, we just need to check numerically the scalar inequality (15) for $P=\tilde{P} / q$ and $k=\tilde{P}^{-1} \tilde{y}$. Remark, for $\mu \in(0,0.5]$ the lefthand side of the inequality (15) is continuously differentiable with respect to $\lambda$. The inequality can be easily checked, for example, on a grid with the sufficiently small step size, i.e. $\lambda=\lambda^{*} \frac{j}{N}, j=0,1,2, \ldots, N$, where $N$ is sufficiently large.

\section{EXAMPLES}

\section{A. First order differentiator}

Consider the first order homogeneous differentiator ( $n=$ 2 ) and design its parameters according the scheme (19) for $f_{0}=1$. The obtained parameters are $\delta=0.0492$,

$$
P=\left(\begin{array}{cc}
20.0608 & -2.6724 \\
-2.6724 & 0.3744
\end{array}\right), k=\left(\begin{array}{l}
-14.0872 \\
-52.2407
\end{array}\right)
$$

for $\alpha=7, \beta=6.9, \mu=0.5$. The inequality (15) has been checked numerically on the grid, which is constructed on the interval $\left[0, \lambda^{*}\right]=[0,2.7484]$ with the step 0.001 .

The simulation results are presented on the Fig. 2 for the reference signal $\xi(t)=5 t+\sin (t)+1$. This test signal has been taken from the paper [7]. The simulations have been done using explicit Euler method with the sampling period 0.01 .

The Fig. 3 depicts the simulation result for the same reference signal, which is measured with a noise. The noise is generated as a sequence of pseudorandom values drawn from the uniform distribution on the open interval $(-0.01,0.01)$. They are applied at the sampled time instances. 


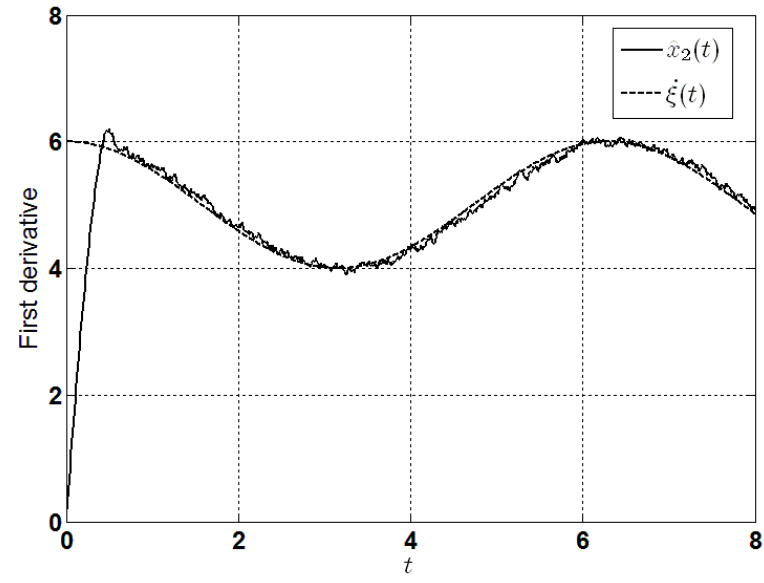

Fig. 3. The first derivative of the noised reference signal

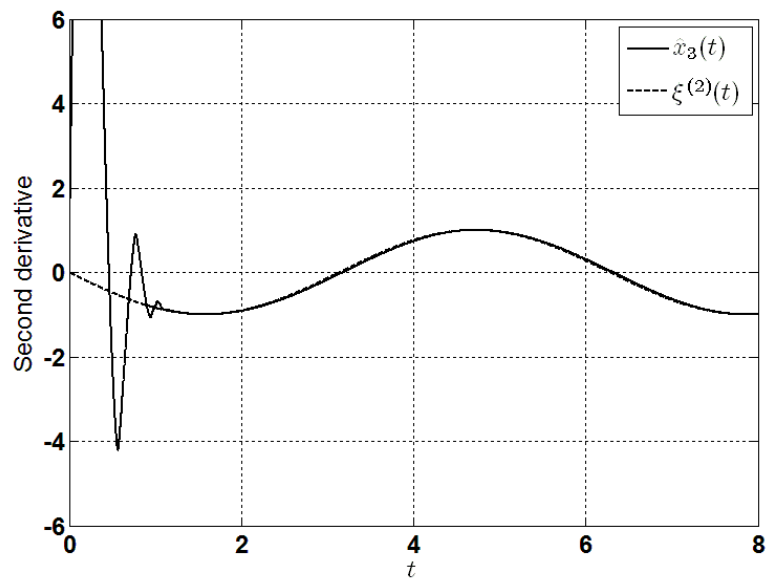

Fig. 4. The second derivative of the reference signal

\section{B. Second order differentiator}

Consider now the second order differentiator $(n=3)$ with parameters $\mu=1 / 3$ and $f_{0}=1$.

The optimization procedure (19) for designing of the gains gives $\delta=0.2559$,

$$
\begin{gathered}
P=\left(\begin{array}{ccc}
24.2073 & -2.8286 & 0.3115 \\
-2.8286 & 0.5764 & -0.0904 \\
0.3115 & -0.0904 & 0.0184
\end{array}\right), \\
k=\left(\begin{array}{c}
-14.7092 \\
-126.0868 \\
-382.7368
\end{array}\right)
\end{gathered}
$$

for $\alpha=5$ and $\beta=4.8$.

The Fig. 4 and 5 present the simulation results for differentiation of the same signal $\xi(t)=5 t+\sin (t)+1$ for the noise-free and noised measurements, respectively. The sampling period for numerical modeling equals to $10^{-3}$.

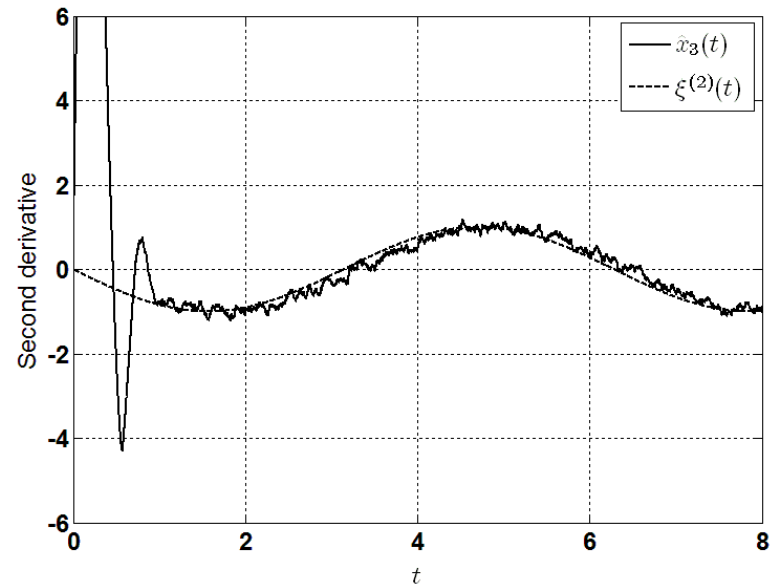

Fig. 5. The second derivative of the noised reference signal

\section{CONCLUSION}

The ILF method for finite-time stability analysis of the homogeneous differentiator is developed. The homogeneous ILF with ellipsoidal level lines is applied for this purpose. The simple scheme for tuning of the observer parameters has been developed for the case of the noise-free measurements.

The optimal tuning of the differentiator parameters for the noised or/and sampled measurements using the ILF approach, and a comparison of the homogeneous differentiator with the high-gain [2] and the HOSM [8] differentiators can be considered as the problems for a future research.

\section{APPENDIX}

\section{A. Proof of Theorem 5}

The function $Q(V, x)$ defined by (5) satisfies the conditions $(1)-C 3$ ) of Theorem 4. Indeed, it is continuously differentiable for all $V \in \mathbb{R}_{+}$and $\forall x \in \mathbb{R}^{n}$.

Since $P>0$ then the following chain of inequalities

$$
\frac{\lambda_{\min }(P)\|x\|^{2}}{\max _{i}\left\{V^{2 r_{i}}\right\}} \leq Q(V, x)+1 \leq \frac{\lambda_{\max }(P)\|x\|^{2}}{\min _{i}\left\{V^{2 r_{i}}\right\}}
$$

implies that for any $z \in \mathbb{R}^{n} \backslash\{0\}$ there exist $V^{-} \in \mathbb{R}_{+}$ and $V^{+} \in \mathbb{R}_{+}: Q\left(V^{-}, x\right)<0<Q\left(V^{+}, x\right)$. Moreover, if $Q(V, x)=0$ then the same chain of inequalities gives

$$
\frac{\min _{i}\left\{V^{2 r_{i}}\right\}}{\lambda_{\max }(P)} \leq\|x\|^{2} \leq \frac{\max _{i}\left\{V^{2 r_{i}}\right\}}{\lambda_{\min }(P)} .
$$

Therefore, the condition C3) of Theorem 4 holds.

Since

$\frac{\partial Q}{\partial V}=-\frac{x^{T} D_{r}\left(V^{-1}\right)(\operatorname{diag}(r) P+P \operatorname{diag}(r)) D_{r}\left(V^{-1}\right) x}{V}$,

then

$$
\operatorname{diag}(r) P+P \operatorname{diag}(r)>0
$$

implies $\frac{\partial Q}{\partial V}<0$ for $\forall V \in \mathbb{R}_{+}$and $x \in \mathbb{R}^{n} \backslash\{0\}$. So, the condition C4) of Theorem 4 also holds. 
We conclude that the equation $Q(V, x)=0$ implicitly defines a proper positive definite function $V: \mathbb{R}^{n} \rightarrow \mathbb{R}_{+} \cup$ $\{0\}$ such that for any $x \in \mathbb{R}^{n}$ we have $Q(V(x), x)=0$.

Let us calculate the time derivative of the function $V$ along the trajectories of the system (3):

$$
\begin{gathered}
\left.\frac{d V}{d t}\right|_{(3)}=-\left[\frac{\partial Q}{\partial V}\right]^{-1} \frac{\partial Q}{\partial x} f(x)= \\
-\left[\frac{\partial Q}{\partial V}\right]^{-1}\left(y^{T} P D_{r}\left(V^{-1}\right) f(x)+f^{T}(x) D_{r}\left(V^{-1}\right) P y\right),
\end{gathered}
$$

where $y=D_{r}\left(V^{-1}\right) x$. The vector field $f$ is homogeneous, i.e. $\lambda^{-m} D_{r}^{-1}(\lambda) f\left(D_{r}(\lambda) x\right)=f(x), \forall \lambda \in \mathbb{R}_{+}$and $\forall x \in \mathbb{R}^{n}$, where $m \in \mathbb{R}$ is the degree of homogeneity. Then

$$
\begin{aligned}
\left.\frac{d V}{d t}\right|_{(3)}= & -\left[\frac{\partial Q}{\partial V}\right]^{-1} V^{m}\left(y^{T} P f(y)+f^{T}(y) P y\right)= \\
& \frac{y^{T} P f(y)+f^{T}(y) P y}{y^{T}(\operatorname{diag}(r) P+P \operatorname{diag}(r)) y} V^{1+m} .
\end{aligned}
$$

Since $Q(V, x)=0$ then $y^{T} P y=1$. The condition 3) and the inequality (6) imply

$$
\begin{gathered}
\left.\frac{d V}{d t}\right|_{(3)} \leq \frac{-\alpha V^{1+m}}{y^{T}(\operatorname{diag}(r) P+P \operatorname{diag}(r)) y}= \\
\frac{-\alpha V^{1+m}}{y^{T} P^{\frac{1}{2}}\left(P^{-\frac{1}{2}} \operatorname{diag}(r) P^{\frac{1}{2}}+P^{\frac{1}{2}} \operatorname{diag}(r) P^{-\frac{1}{2}}\right) P^{\frac{1}{2}} y} \leq \\
\frac{-\alpha V^{1+m}}{\gamma y^{T} P y}=-\frac{\alpha}{\gamma} V^{1+m},
\end{gathered}
$$

where the constants $\alpha$ and $\gamma$ are defined in the statement of the theorem.

Obviously, if $m<0$ then the designed ILF satisfies the conditions of Theorem 4 about finite-time stability. For $m=$ 0 we have exponential stablity: $V(t) \leq V_{0} e^{-\frac{\alpha}{\gamma} t}$

Let us show that the implicitly defined Lyapunov function $V(x)$ is homogeneous of degree 1 with the same weights $r$, i.e. $V\left(D_{r}(\lambda) x\right)=\lambda V(x)$ for all $\lambda \in \mathbb{R}_{+}$and $x \in \mathbb{R}^{n}$. Indeed, since $Q\left(V, D_{r}(\lambda) x\right)=Q(V / \lambda, x)$ then

$$
\begin{gathered}
Q\left(V\left(D_{r}(\lambda) x\right), D_{r}(\lambda) x\right)= \\
Q\left(\lambda^{-1} V\left(D_{r}(\lambda) x\right), x\right)=0=Q(V(x), x),
\end{gathered}
$$

i.e $\lambda^{-1} V\left(D_{r}(\lambda) x\right)=V(x)$.

\section{REFERENCES}

[1] C. L. Smith, Practical process control. New Jersey: John Wiley \& Sons, 2009.

[2] H. K. Khalil, "High-gain observers in nonlinear feedback control," Lecture Notes in Control and Information Sciences, vol. 244, pp. 249268, 1999.

[3] T. Menard, E. Moulay, and W. Perruquetti, "A global high-gain finitetime observer," IEEE Transactions on Automatic Control, vol. 55(6), pp. 1500-1506, 2010.

[4] R. Patton and J. Chen, "Observer-based fault detection and isolation: Robustness and applications," Control Engineering Practice, vol. 5(5), pp. 671-682, 1997.

[5] C. Chen and J. Lee, "Design of high-order digital differentiators using $l_{1}$ error criteria," IEEE Trans. Circuits Syst. II, vol. 42, no. 4, pp. 287291, Avril 1995.
[6] M. Hanke and O. Scherzer, "Error analysis of an equation error method for the identification of of the diffusion coefficient in a quasi-linear parabolic differentical equation," SIAM J. Appl. Math., vol. 59, no. 3, pp. 1012-1027, 1999.

[7] A. Levant, "Robust exact differentiation via sliding mode technique," Automatica, vol. 34 (3), pp. 379-384, 1998.

[8] —, "Higher-order sliding modes, differentiation and outputfeedback control," International Journal of Control, vol. 76 (9/10), pp. 924-941, 2003.

[9] W. Perruquetti, T. Floquet, and E. Moulay, "Finite-time observers: Application to secure communication," IEEE Transactions on Automatic Control, vol. 53(1), pp. 356-360, 2008.

[10] R. Sanfelice and L. Praly, "On the performance of high-gain observers with sign-indefinite gain adaptation under measurement noise," Automatica, vol. 47(10), pp. 2141-2330, 2011.

[11] V. Korobov, "A general approach to synthesis problem," Doklady Academii Nauk SSSR, vol. 248, pp. 1051-1063, 1979.

[12] J. Adamy and A. Flemming, "Soft variable-structure controls: a survey," Automatica, vol. 40, pp. 1821-1844, 2004.

[13] A. Polyakov, D. Efimov, and W. Perruquetti, "Finite-time stabilization using implicit lyapunov function technique,", in 9th Symposium on Nonlinear Control Systems, Toulouse, France,, 4-6 September 2013, pp. $140-145$.

[14] E. Roxin, "On finite stability in control systems," Rendiconti del Circolo Matematico di Palermo, vol. 15(3), pp. 273-283, 1966.

[15] S. Bhat and D. Bernstein, "Finite-time stability of continuous autonomous systems," SIAM Journal of Control and Optimization, vol. 38(3), pp. 751-766, 2000.

[16] V. Zubov, "On systems of ordinary differential equations with generalized homogenous right-hand sides," Izvestia vuzov. Mathematica., vol. 1, pp. 80-88, 1958 (in Russian).

[17] S. P. Bhat and D. S. Bernstein, "Geometric homogeneity with applications to finite-time stability," Mathematics of Control, Signals and Systems, vol. 17, pp. 101-127, 2005.

[18] R. Courant and F. John, Introduction to calculus and analysis (Vol. II/1). New York: Springer, 2000.

[19] H. Nakamura, N. Nakamura, and H. Nishitani, "Stabilization of homogeneous systems using implicit control lyhapunov functions," in 7th IFAC Symposium on Nonlinear Control Systems, Pretoria, Shouth Africa, 21-24 August 2007, pp. 561-566.

[20] H. Tuan, P. Apkaryan, and H. Tuy, "Advanced global optimization algorithms for parameterized lmis," in 38th Conference on Decision and Control, Pheonix, Arizona, USA, 7-10 December 1999. 